\title{
MOLECULAR GAS EXCITATION IN NGC 253
}

\author{
W. F. WALL ${ }^{1}$, D. T. JAFFe, ${ }^{1}$ F. P. ISRAEL, ${ }^{2}$ AND F. N. BASH ${ }^{1}$ \\ Received 1991 January 24 ; accepted 1991 May 2
}

\begin{abstract}
We have observed the $J=3 \rightarrow 2$ and $J=2 \rightarrow 1$ lines of ${ }^{13} \mathrm{CO}$ and ${ }^{12} \mathrm{CO}$ with $22^{\prime \prime}-24^{\prime \prime}$ resolution in the central region of the spiral galaxy NGC 253 . The ${ }^{13} \mathrm{CO} J=3 \rightarrow 2$ emission is pointlike $\left(\lesssim 15^{\prime \prime}\right)$, whereas the emission in the other CO lines is consistent with emission from a $\sim 40^{\prime \prime} \times 10^{\prime \prime} \mathrm{FWHM}$ bar. The ratio of ${ }^{13} \mathrm{CO}$ $J=3 \rightarrow 2$ to ${ }^{13} \mathrm{CO} J=2 \rightarrow 1$ line intensities toward the nucleus requires warm $\left(T_{K} \gtrsim 100 \mathrm{~K}\right)$, dense $\left[n\left(\mathrm{H}_{2}\right) \gtrsim\right.$ $10^{4}-10^{5} \mathrm{~cm}^{-3}$ ] molecular gas. Spatial coincidence of $3 \mathrm{~mm}$ continuum and [Ne $\left.\mathrm{II}\right] 12.8 \mu \mathrm{m}$ emission from $\mathrm{H}$ II regions and mid-IR emission from hot dust with the ${ }^{13} \mathrm{CO} J=3 \rightarrow 2$ emission implies a common source of excitation, possibly related to the intense star formation that has occurred in the nucleus. The decline in the ${ }^{13} \mathrm{CO} J=3 \rightarrow 2$ intensity with distance from the nucleus reflects a decline in both density and temperature. The declining density, for the bulk of the $\mathrm{CO}$ line-emitting gas, is inferred from the drop in the ${ }^{13} \mathrm{CO}$ $J=3 \rightarrow 2 /{ }^{13} \mathrm{CO} J=2 \rightarrow 1$ intensity ratio, while the small ${ }^{13} \mathrm{CO} J=3 \rightarrow 2$ source size compared to the size of the emission region for lines of high dipole moment molecules (e.g., CS $J=2 \rightarrow 1$ ) implies a decline in gas temperature. A drop in the ${ }^{12} \mathrm{CO} J=3 \rightarrow 2 / J=2 \rightarrow 1$ intensity ratio indicates that the molecular gas density is low $30^{\prime \prime}$ from the nucleus. The molecular gas mass in the central $50^{\prime \prime}$, estimated from the optically thin lines of ${ }^{13} \mathrm{CO}$, is $\sim 4 \times 10^{7} \mathrm{M}_{\odot}$.
\end{abstract}

Subject headings: galaxies: individual (NGC 253) - galaxies: interstellar matter - interstellar: molecules

\section{INTRODUCTION}

NGC 253 is a nearby $(D=2.5 \mathrm{Mpc}$; de Vaucouleurs 1978 ; see also Davidge \& Pritchett 1990), highly inclined (see Table 1 for adopted parameters) Sc galaxy undergoing a starburst (Wynn-Williams et al. 1979; Rieke et al. 1980). It has a $240^{\prime \prime}$ long stellar bar (Scoville et al. 1985) and high-resolution millimeter-wave observations show that a $40^{\prime \prime} \times 10^{\prime \prime}$ FWHM barlike feature dominates the ${ }^{12} \mathrm{CO} J=1 \rightarrow 0$ emission (Canzian, Mundy, \& Scoville 1988) from the central region. In addition to $\mathrm{CO}$, lines of other, rarer molecules such as $\mathrm{CS}$, HCN, and $\mathrm{HCO}^{+}$(Rickard et al. 1977b; Stark \& Wolff 1919; Mauersberger \& Henkel 1989; Rieu, Nakai, \& Jackson 1989), have been detected and suggest that the central $30^{\prime \prime}-40^{\prime \prime}$ diameter of NGC 253 contains dense $\left[n\left(\mathrm{H}_{2}\right) \geq 10^{5} \mathrm{~cm}^{-3}\right]$ molecular gas. Multiline observations of $\mathrm{HC}_{3} \mathrm{~N}$ (Mauersberger, Henkel, \& Sage 1990) show that at least some of this dense gas is warm $\left(T_{K} \geq 60 \mathrm{~K}\right)$.

To explore further the density and temperature structure in the molecular gas in NGC 253, we have observed the $J=$ $3 \rightarrow 2$ and $J=2 \rightarrow 1$ lines of ${ }^{13} \mathrm{CO}$ and ${ }^{12} \mathrm{CO}$ - all with $20^{\prime \prime}-24^{\prime \prime}$ resolution. $\mathrm{CO}$ is an ideal probe of the interstellar molecular gas in galaxies. It is abundant and sturdy enough to be present almost everywhere hydrogen is in molecular form. The small dipole moment of $\mathrm{CO}$ makes its lowest rotational transition relatively easy to thermalize. Beam-matched observations of different rotational lines of ${ }^{12} \mathrm{CO}$ and especially ${ }^{13} \mathrm{CO}$ provide the best method for determining the excitation conditions in the bulk of the molecular gas. A higher $J$ transition of $\mathrm{CO}$ requires higher gas density to thermalize and varies in brightness more rapidly with temperature than does a low $J$ transition, making lines arising in different rotational levels sensitive to different ranges of densities and temperatures. Hence, comparing the strengths of the different lines

\footnotetext{
Astronomy Department, University of Texas at Austin, Austin, TX 78712 1083

${ }^{2}$ Sterrewacht, Postbus 9513, 2300RA, Leiden, Netherlands
}

provides estimates of molecular gas excitation. Since the ${ }^{13} \mathrm{CO}$ lines are less optically thick than the corresponding ${ }^{12} \mathrm{CO}$ lines, they probe conditions integrated over a larger fraction of the molecular material. They are also more sensitive than ${ }^{12} \mathrm{CO}$ lines to the physical conditions in the molecular gas because trapping effects are not as important for less opaque lines. The ${ }^{13} \mathrm{CO}$ line data, together with the ${ }^{12} \mathrm{CO}$ data, will allow us to form a picture of the density and temperature structure of the molecular gas in the inner region of NGC 253.

As a nearby, infrared-bright galaxy, NGC 253 is similar in a number of ways to M82. Their central regions have comparable far-IR luminosities (M82: $3 \times 10^{10} L_{\odot}$ in $\sim 400$ pc diameter; NGC $253: 1.5 \times 10^{10} L_{\odot}$ in $\sim 400$ pc diameter, Telesco \& Harper 1980), and similar ${ }^{12} \mathrm{CO} J=1 \rightarrow 0$ luminosities $\left(5 \times 10^{3} L_{\odot}\right.$ in $\sim 700$ pc diameter for both galaxies; Rickard et al. 1977a; Young \& Scoville 1984; Scoville et al. 1985). Further observational comparisons of these two galaxies can provide insight into the influence of the starburst phenomenon on conditions in the molecular interstellar medium in the central kiloparsec.

\section{OBSERVATIONS}

We made the ${ }^{13} \mathrm{CO}$ and ${ }^{12} \mathrm{CO} J=3 \rightarrow 2(v=330.6$ and $345.8 \mathrm{GHz}$ respectively) observations at the Caltech Submillimeter Observatory (CSO) $10 \mathrm{~m}$ telescope with the facility SIS receiver (Ellison, Schaffer, \& Schaal 1989). On 1989 November 7, the night of the ${ }^{13} \mathrm{CO} J=3 \rightarrow 2$ observations, the receiver temperature, $T_{\mathrm{rx}}$, was $570 \mathrm{~K}$ (SSB) and the atmospheric opacity was 0.25 at the zenith. When observing the 331 $\mathrm{GHz}^{13} \mathrm{CO} J=3 \rightarrow 2$ line, a strong telluric $\mathrm{H}_{2} \mathrm{O}$ line near 325 $\mathrm{GHz}$ causes the atmospheric opacity in the signal and image sidebands to differ. Atmospheric modeling shows that, for our lower sideband observations with $v_{\mathrm{IF}}=1.4 \mathrm{GHz}$, the correction to the standard chopper wheel calibration is $10 \%$ or less. We have not applied such a correction. The ${ }^{12} \mathrm{CO} J=3 \rightarrow 2$ line was observed 1989 June 12 when $T_{\mathrm{rx}}$ was $480 \mathrm{~K}$ (SSB). Both $J=3 \rightarrow 2$ line observations had beam sizes of $22^{\prime \prime}-24^{\prime \prime}$ 
TABLE 1

AdOPTED PARAMETERS FOR NGC 253

\begin{tabular}{|c|c|}
\hline Parameter & Value \\
\hline$\alpha(1950)^{\mathbf{a}}$ & $00^{\mathrm{h}} 45^{\mathrm{m}} 05^{\mathrm{s}} .8$ \\
\hline$\delta(1950)^{\mathbf{a}} .$. & $-25^{\circ} 33^{\prime} 38^{\prime \prime}$ \\
\hline Distance $^{b} \ldots$ & $2.5 \mathrm{Mpc}$ \\
\hline Inclination $^{c} \ldots \ldots \ldots \ldots \ldots \ldots$ & 78.5 \\
\hline Position angle of major axis ${ }^{c} \ldots \ldots \ldots \ldots \ldots$ & $51^{\circ}$ \\
\hline Beam size on galaxy $\left(24^{\prime \prime}\right.$ FWHM $) \ldots \ldots \ldots \ldots$ & $290 \mathrm{pc}$ \\
\hline
\end{tabular}

${ }^{a}$ Becklin, Fomalont, \& Neugebauer 1973.

b de Vaucouleurs 1978

c Pence 1981.

(FWHM) and a main beam efficiency $\left(\eta_{\mathrm{MB}}=T_{A}^{*} / T_{\mathrm{MB}}\right.$, where $T_{A}^{*}$ is the antenna temperature corrected for atmospheric losses and losses due to spillover and scattering and where $T_{\mathrm{MB}}$ is the radiation temperature of a uniform source filling the antenna beam to the first null) of 0.5 as derived from measurements of Jupiter, assuming $T_{\text {disk }}=170 \mathrm{~K}$ (Hildebrand et al. 1985). The observations were position-switched (off-positions $30^{\prime}$ to the east and west in azimuth) and the tracking was accurate to $3^{\prime \prime}-4^{\prime \prime}$. Since the absolute pointing was more uncertain, the peak of the $J=3 \rightarrow 2$ emission, assumed to be the $2 \mu \mathrm{m}$ peak (Becklin, Fomalont, \& Neugebauer 1973), was chosen as the $(0,0)$ position.

The $J=2 \rightarrow 1$ lines of ${ }^{13} \mathrm{CO}$ and ${ }^{12} \mathrm{CO}(v=220.4$ and 230.5 $\mathrm{GHz}$, respectively) were observed at five positions with the James Clerk Maxwell Telescope (JCMT) $15 \mathrm{~m}$ antenna on 1988 August 9-10. The Schottky receiver had $T_{\mathrm{rx}}=800-900 \mathrm{~K}$ (SSB) and the zenith atmospheric opacity ranged from 0.05 to 0.1 . The beam size was $21^{\prime \prime}$ (FWHM) and $\eta_{\mathrm{MB}}$ was 0.6 as derived from measurements of Mars, assuming $T_{\text {disk }}=210 \mathrm{~K}$ (see Wright 1976). Position-switching was used to obtain the spectra and the off-position was $27^{\prime} \mathrm{E}$. The pointing was accurate to within $5^{\prime \prime}-7^{\prime \prime}$.

To improve spatial coverage of the ${ }^{13} \mathrm{CO}$ and ${ }^{12} \mathrm{CO}$ $J=2 \rightarrow 1$ data, these lines were also observed with the Swedish ESO Submillimetre Telescope (SEST) $15 \mathrm{~m}$ antenna during 1989 July $1-12$. The observations were carried out with a Schottky receiver where $T_{\mathrm{rx}}=700-800 \mathrm{~K}$ (SSB). The beam size was $24^{\prime \prime}$ (FWHM), the atmospheric opacity remained near 0.2 , and $\eta_{\mathrm{MB}}$, as derived from Jupiter measurements, was 0.5 . The SEST observations were beam-switched (i.e., using a chopping secondary), where the on and off beams were separated by $11^{\prime} 37^{\prime \prime}$. The absolute pointing uncertainty was $10^{\prime \prime}$, but the tracking was better than $5^{\prime \prime}$ and was checked by repeatedly observing the ${ }^{12} \mathrm{CO}$ peak position chosen as the $(0,0)$ position. As a result, the pointing of the SEST data is within 5" of those of the CSO positions, as seen from the general agreement among the various line profiles for each position (see Figs. 3 and 4 below). Because the absolute pointing of the JCMT observations is better than $5^{\prime \prime}-7$ ", it follows that the SEST and CSO observations are centered within $5^{\prime \prime}-10^{\prime \prime}$ of the $2 \mu \mathrm{m}$ nucleus (Becklin et al. 1973). Additional observations with the SEST were made of the ${ }^{12} \mathrm{CO} J=1 \rightarrow 0$ line $(v=115.3 \mathrm{GHz})$ with a 43" (FWHM) beam on 1989 July 4 . The $T_{\text {rx }}$ was $290 \mathrm{~K}$ (SSB) and the opacity of the atmosphere was 0.3 .

All lines are calibrated in terms of main-beam radiation temperature, $T_{\mathrm{MB}}$. All observed positions are along the major and minor axes. Table 1 lists the equatorial coordinates for position $(0,0)$ and other adopted parameters for NGC 253. The various line parameters - peak $T_{\mathrm{MB}}$, integrated line strength,
TABLE 2

NGC $253 \mathrm{CO} J=3 \rightarrow 2$ Line Parameters

\begin{tabular}{|c|c|c|c|c|c|c|}
\hline \multicolumn{2}{|c|}{ Position } & \multirow{2}{*}{$\begin{array}{c}T_{\mathrm{MB}}(\text { Peak }) \\
(\mathrm{K})\end{array}$} & \multirow{2}{*}{$\int_{\left(\mathrm{K} \mathrm{km} \mathrm{s}^{-1}\right)} T_{\mathrm{MB}} d V$} & \multirow{2}{*}{$\begin{array}{l}V_{\text {peak }} \\
\left(\mathrm{km} \mathrm{s}^{-1}\right)\end{array}$} & \multirow{2}{*}{$\begin{array}{l}V_{\text {mean }} \\
\left(\mathrm{km} \mathrm{s}^{-1}\right)\end{array}$} & \multirow{2}{*}{$\begin{array}{l}\Delta V_{\left(\mathrm{FWHM}_{\mathrm{M}}\right.} \\
\left(\mathrm{km} \mathrm{s}^{-1}\right)\end{array}$} \\
\hline$\Delta \alpha^{\prime \prime}$ & $\Delta \delta^{\prime \prime}$ & & & & & \\
\hline \multicolumn{7}{|c|}{${ }^{12} \mathrm{CO} J=3 \rightarrow 2$} \\
\hline 24 , & 18 & 1.4 & 172 & 160 & 183 & $\sim 105$ \\
\hline 16 , & 12 & 3.4 & 518 & 160 & 189 & 120 \\
\hline 8 & 8 & 4.8 & 900 & 195 & 221 & 195 \\
\hline 0 , & 0 & 6.2 & 1194 & 290 & 267 & 195 \\
\hline-6 & -6 & 5.4 & 842 & 305 & 300 & 140 \\
\hline-16 & -12 & 3.6 & 494 & 315 & 311 & 95 \\
\hline-24 & -18 & 1.2 & 168 & 305 & 295 & 75 \\
\hline-32 , & -24 & 1.0 & 164 & $\sim 320$ & 310 & $\sim 140$ \\
\hline 6 & -8 & 4.0 & 700 & 305 & 282 & 167 \\
\hline-6 & 8 & 3.6 & 648 & 280 & 267 & 175 \\
\hline-12 , & 16 & 1.1 & 190 & $\sim 280$ & 262 & $\sim 145$ \\
\hline \multicolumn{7}{|c|}{${ }^{13} \mathrm{CO} J=3 \rightarrow 2$} \\
\hline 9, & 7 & 0.32 & 22 & 150 & 180 & 100 \\
\hline 0 & 0 & 1.0 & 210 & 170 & 198 & 210 \\
\hline-9 , & -7 & 0.60 & 79 & 280 & 285 & 70 \\
\hline 7 & -9 & $\sim 0.2$ & $\sim 37$ & $\sim 210$ & $\sim 250$ & $\sim 150$ \\
\hline-7 , & 9 & $\sim 0.3$ & $\sim 65$ & $\sim 200$ & $\sim 200$ & $\sim 150$ \\
\hline
\end{tabular}

velocity widths, etc. - are listed in Tables 2,3 , and 4 . The spectra are shown in Figures 1, 2, 3, and 4. Because on average, the JCMT ${ }^{12} \mathrm{CO} J=2 \rightarrow 1$ lines are stronger than the corresponding SEST lines, the ${ }^{12} \mathrm{CO} J=2 \rightarrow 1$ line strengths have

TABLE 3

NGC 253 CO $J=2 \rightarrow 1$ Line Parameters

\begin{tabular}{|c|c|c|c|c|c|c|}
\hline \multicolumn{2}{|c|}{ Position } & \multirow{2}{*}{$\begin{array}{c}T_{\mathrm{MB}}(\text { Peak }) \\
(\mathrm{K})\end{array}$} & \multirow{2}{*}{$\underset{\left(\mathrm{K} \mathrm{km} \mathrm{s}^{-1}\right)}{\int_{\mathrm{MB}} d V}$} & \multirow{2}{*}{$\underset{\left(\mathrm{km} \mathrm{s}^{-1}\right)}{V_{\text {peak }}}$} & \multirow{2}{*}{$\begin{array}{c}V_{\text {mean }} \\
\left(\mathrm{km} \mathrm{s}^{-1}\right)\end{array}$} & \multirow{2}{*}{$\begin{array}{l}\Delta V_{\text {(FWHM) }} \\
\left(\mathrm{km} \mathrm{s}^{-1}\right)\end{array}$} \\
\hline$\Delta \alpha^{\prime \prime}$ & $\Delta \delta^{\prime \prime}$ & & & & & \\
\hline \multicolumn{7}{|c|}{${ }^{12} \mathrm{CO} J=2 \rightarrow 1$} \\
\hline 32 , & 24 & 2.0 & 300 & 160 & 169 & 150 \\
\hline 32 , & $24^{\mathrm{a}}$ & $1.2^{\mathrm{a}}$ & $203^{a}$ & $153^{\mathrm{a}}$ & $145^{\mathrm{a}}$ & $180^{\mathrm{a}}$ \\
\hline 24 , & 18 & 3.5 & 605 & 160 & 188 & 165 \\
\hline 16 , & 12 & 3.9 & 896 & 165 & 217 & 220 \\
\hline 16 , & $12^{\mathrm{a}}$ & $5.1^{\mathrm{a}}$ & $1029^{\mathrm{a}}$ & $160^{\mathrm{a}}$ & $181^{\mathrm{a}}$ & $180^{\mathrm{a}}$ \\
\hline 8 & 6 & 4.0 & 949 & 265 & 239 & 225 \\
\hline 8 , & $6^{\mathrm{a}}$ & $4.0^{\mathrm{a}}$ & $894^{\mathrm{a}}$ & $270^{\mathrm{a}}$ & $236^{\mathrm{a}}$ & $210^{\mathrm{a}}$ \\
\hline 0 , & 0 & 5.0 & 926 & 310 & 290 & 155 \\
\hline 0 & $0^{\mathrm{a}}$ & $6.1^{\mathrm{a}}$ & $1152^{\mathrm{a}}$ & $285^{\mathrm{a}}$ & $270^{\mathrm{a}}$ & $170^{\mathrm{a}}$ \\
\hline-8 & -6 & 4.5 & 763 & 315 & 304 & 145 \\
\hline-16 & -12 & 2.4 & 333 & 310 & 316 & 115 \\
\hline-16 & $-12^{\mathrm{a}}$ & $2.2^{\mathrm{a}}$ & $330^{\mathrm{a}}$ & $315^{\mathrm{a}}$ & $318^{\mathrm{a}}$ & $160^{\mathrm{a}}$ \\
\hline-24 , & -18 & 1.3 & 206 & 320 & 321 & 100 \\
\hline-32 , & -24 & 0.7 & 75 & 320 & 331 & 150 \\
\hline 12 , & -16 & 1.0 & 218 & 310 & 295 & 195 \\
\hline 6 & -8 & 3.9 & 829 & 290 & 276 & 200 \\
\hline-6 & 8 & 2.7 & 536 & 280 & 267 & 190 \\
\hline-12 & 16 & 1.6 & 338 & 300 & 276 & 180 \\
\hline-18 & 24 & 0.7 & 109 & $\sim 250$ & 251 & 180 \\
\hline \multicolumn{7}{|c|}{${ }^{13} \mathrm{CO} J=2 \rightarrow 1$} \\
\hline 16 , & 12 & 0.35 & 71 & 170 & 187 & 220 \\
\hline 6 & 6 & 0.35 & 67 & 150 & 205 & 220 \\
\hline & 0 & 0.50 & 104 & 260 & 245 & 170 \\
\hline 0 & $0^{\mathrm{a}}$ & $\sim 0.4^{\mathrm{a}}$ & $\sim 75^{\mathrm{a}}$ & $\sim 220^{\mathrm{a}}$ & $\sim 230^{\mathrm{a}}$ & $\sim 180^{\mathrm{a}}$ \\
\hline-10 & -6 & 0.47 & 69 & 275 & 268 & 110 \\
\hline 6 & -8 & 0.27 & 53 & 270 & 253 & 210 \\
\hline-6 & 8 & 0.52 & 88 & 270 & 257 & 185 \\
\hline-12 & 16 & 0.20 & 26 & 290 & 258 & 130 \\
\hline
\end{tabular}

a JCMT data. 


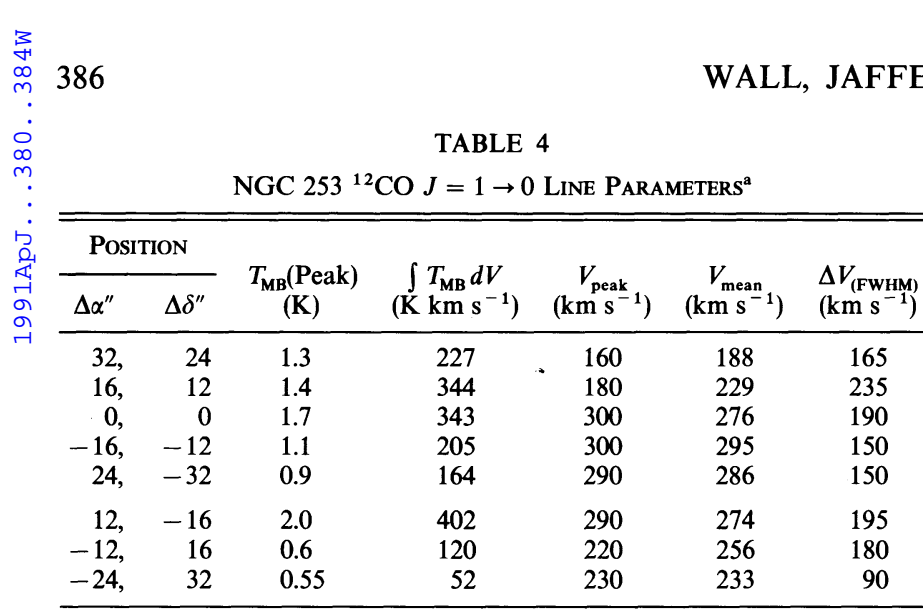

a $43^{\prime \prime}$ beam.

been adjusted downward by $10 \%$ for the JCMT data and upward by $12 \%$ for the SEST data. Even though the absolute calibration uncertainty is roughly $\pm 20 \%$, the position-to-position calibration uncertainty for observations of a single line is about $\pm 10 \%$; spatial variations of line ratios are then more reliable than the actual values of those ratios. The line ratios, themselves, have a $\pm 30 \%$ uncertainty.

\section{RESULTS}

Figures 1 and 2 show the ${ }^{13} \mathrm{CO}$ and ${ }^{12} \mathrm{CO}$ spectra toward $(0,0)$, and Figures 3 and 4 show the ${ }^{13} \mathrm{CO}$ and ${ }^{12} \mathrm{CO}$ spectra toward the major and minor axis positions. The line strengths and velocities are listed in Tables 2,3 , and 4 . At the $(0,0)$ position, the ${ }^{12} \mathrm{CO}$ line strength increases from $J=1 \rightarrow 0$ to $J=2 \rightarrow 1$ and remains constant from $J=2 \rightarrow 1$ to $J=3 \rightarrow 2$. In contrast, the ${ }^{13} \mathrm{CO} J=3 \rightarrow 2$ line is significantly stronger than the ${ }^{13} \mathrm{CO} J=2 \rightarrow 1$ line. The strengths of all lines decrease monotonically from the center.

Differences in the morphology of the ${ }^{13} \mathrm{CO} J=3 \rightarrow 2$ emission from that of the other ${ }^{12} \mathrm{CO}$ and ${ }^{13} \mathrm{CO}$ lines suggest that at least two different components may be present in the molecular gas of NGC 253: nuclear and "bar." The ${ }^{13} \mathrm{CO} J=3 \rightarrow 2$ line strength falls to roughly half its peak value one-half a beam from the center, implying pointlike (source size $\lesssim 15^{\prime \prime}$ ) emission. The ${ }^{12} \mathrm{CO} J=3 \rightarrow 2, J=2 \rightarrow 1, J=1 \rightarrow 0$ as well as

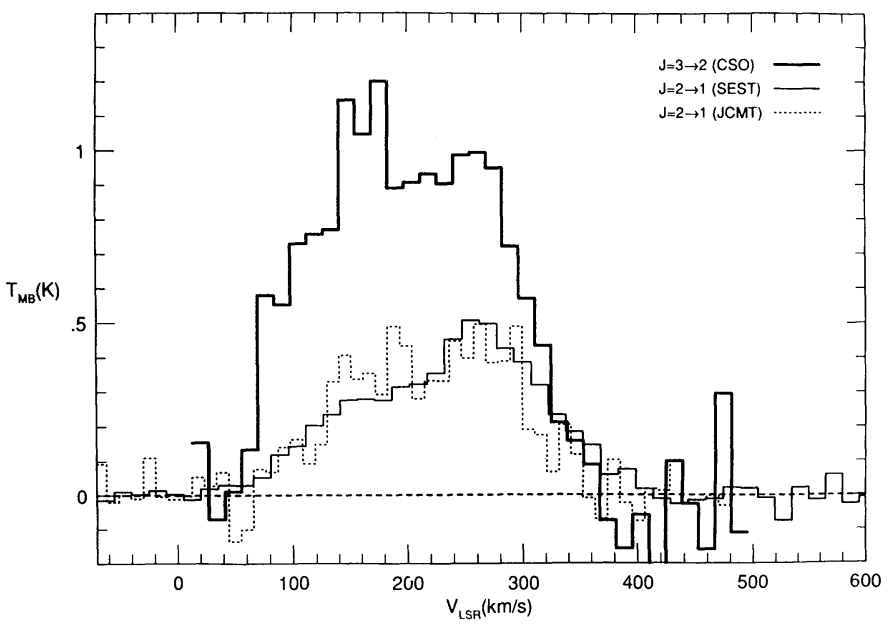

Fig. 1.-The ${ }^{13} \mathrm{CO} J=3 \rightarrow 2$ and $J=2 \rightarrow 1$ spectra toward the center of NGC $253\left[\alpha(1950)=0^{\mathrm{h}} 45^{\mathrm{m}} 5 \mathrm{~s} .8, \delta(1950)=-25^{\circ} 33^{\prime} 38^{\prime \prime}\right]$ are shown above as main-beam radiation temperature, $T_{M B}$, vs. LSR velocity. Both lines were observed with $21^{\prime \prime}-24^{\prime \prime}$ beams.

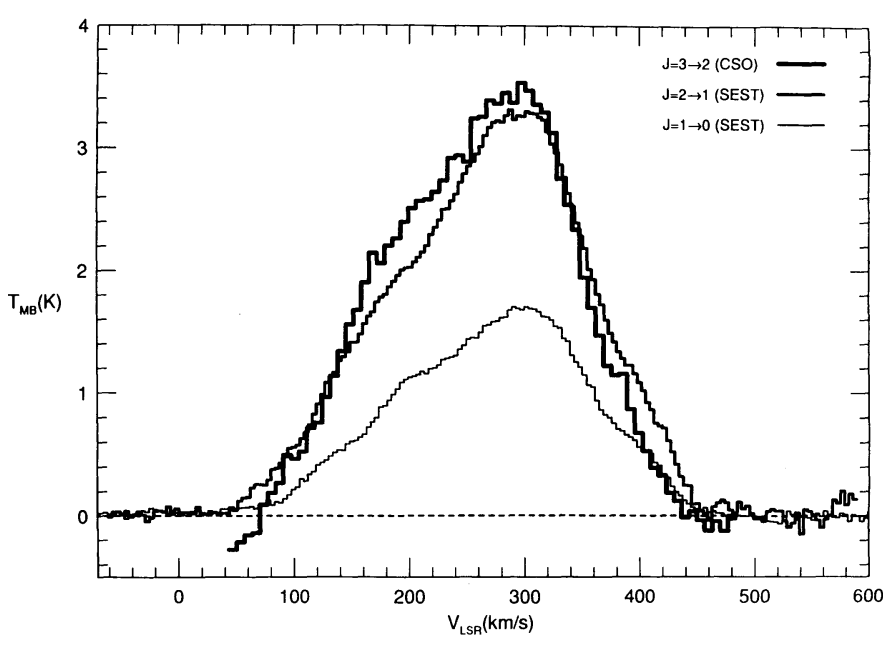

Fig. 2.-The ${ }^{12} \mathrm{CO} J=3 \rightarrow 2, J=2 \rightarrow 1$ and $J=1 \rightarrow 0$ spectra toward the center of NGC 253 are illustrated, where the $J=3 \rightarrow 2$ and $J=2 \rightarrow 1$ lines have been convolved to the $43^{\prime \prime}$ resolution of the $J=1 \rightarrow 0$ line.

${ }^{13} \mathrm{CO} J=2 \rightarrow 1$ distributions, however, are consistent with emission from a 40" $\times 10^{\prime \prime}$ FWHM "bar" (as found by Canzian et al. 1988) convolved with a $20^{\prime \prime}$ beam, although a substantial contribution from a compact nuclear component to the strength of any of these lines cannot be excluded.

There is a large velocity difference between the ${ }^{13} \mathrm{CO}$ $J=3 \rightarrow 2$ and ${ }^{12} \mathrm{CO}$ lines, which provides further evidence that most of the ${ }^{13} \mathrm{CO} J=3 \rightarrow 2$ emission arises in a separate nuclear component. The ${ }^{13} \mathrm{CO} J=3 \rightarrow 2$ mean velocity at $(0,0)$ is $\sim 200 \mathrm{~km} \mathrm{~s}^{-1}, 60-80 \mathrm{~km} \mathrm{~s}^{-1}$ lower (a difference much too large to be a pointing offset) than the ${ }^{12} \mathrm{CO}$ velocities we measured at the central position. The ${ }^{13} \mathrm{CO} J=2 \rightarrow 1$ emission, which has a distribution similar to that of the ${ }^{12} \mathrm{CO}$ lines, must arise in both the nuclear and "bar" components. The intermediate mean velocity of the ${ }^{13} \mathrm{CO} J=2 \rightarrow 1$ line $(\sim 245$ $\mathrm{km} \mathrm{s}^{-1}$ ) may reflect the contribution of the two components to the emission.

For lines other than the ${ }^{13} \mathrm{CO} J=3 \rightarrow 2$, there is good agreement $\left(\leq \pm 10 \mathrm{~km} \mathrm{~s}^{-1}\right)$ between the mean velocities of most of the different transitions at a given position. The ${ }^{12} \mathrm{CO}$ $J=2 \rightarrow 1$ observations from SEST, however, have mean velocities $\sim 20 \mathrm{~km} \mathrm{~s}^{-1}$ higher than those of the other transitions or of the JCMT ${ }^{12} \mathrm{CO} J=2 \rightarrow 1$ lines. The velocity gradient in the central region of NGC 253 is $3-4 \mathrm{~km} \mathrm{~s}^{-1} \operatorname{arcsec}^{-1}$ implying that the SEST ${ }^{12} \mathrm{CO} J=2 \rightarrow 1$ positions are offset by $5^{\prime \prime}-7^{\prime \prime}$ from the positions where other data were taken. A shift of $\sim 6^{\prime \prime}$ to the southwest would bring the SEST ${ }^{12} \mathrm{CO} J=2 \rightarrow 1$ data into agreement with the other observations. A shift of this magnitude has not been applied to the tables or figures and would have little effect on the line ratios at the center. We do, however, shift the data when we calculate and discuss the ratio of the ${ }^{12} \mathrm{CO} J=3 \rightarrow 2$ to ${ }^{12} \mathrm{CO} J=2 \rightarrow 1$ line strengths farther out on the "bar," where it has a material effect on the results.

\subsection{Nucleus}

There is evidence for a warm $\left(T_{K} \gtrsim 100 \mathrm{~K}\right)$, compact $\left(\lesssim 15^{\prime \prime}\right)$ source in the center of NGC 253 . We infer the high temperature from the ratio of ${ }^{13} \mathrm{CO}$ lines, ${ }^{13} R_{32}\left\{\right.$ where ${ }^{x} R_{J^{\prime} J^{\dagger}}=$ $\left.\int T_{\mathrm{MB}}\left[{ }^{x} \mathrm{CO} \quad J^{\prime} \rightarrow\left(J^{\prime}-1\right)\right] d V / \int T_{\mathrm{MB}}\left[{ }^{x} \mathrm{CO} \quad J^{\dagger} \rightarrow\left(J^{\dagger}-1\right)\right] d V\right\}$, which is $\simeq 2$ at the center position (see Tables 2 and 3 ). The pointlike ${ }^{13} \mathrm{CO} J=3 \rightarrow 2$ emission and extended ${ }^{13} \mathrm{CO}$ 


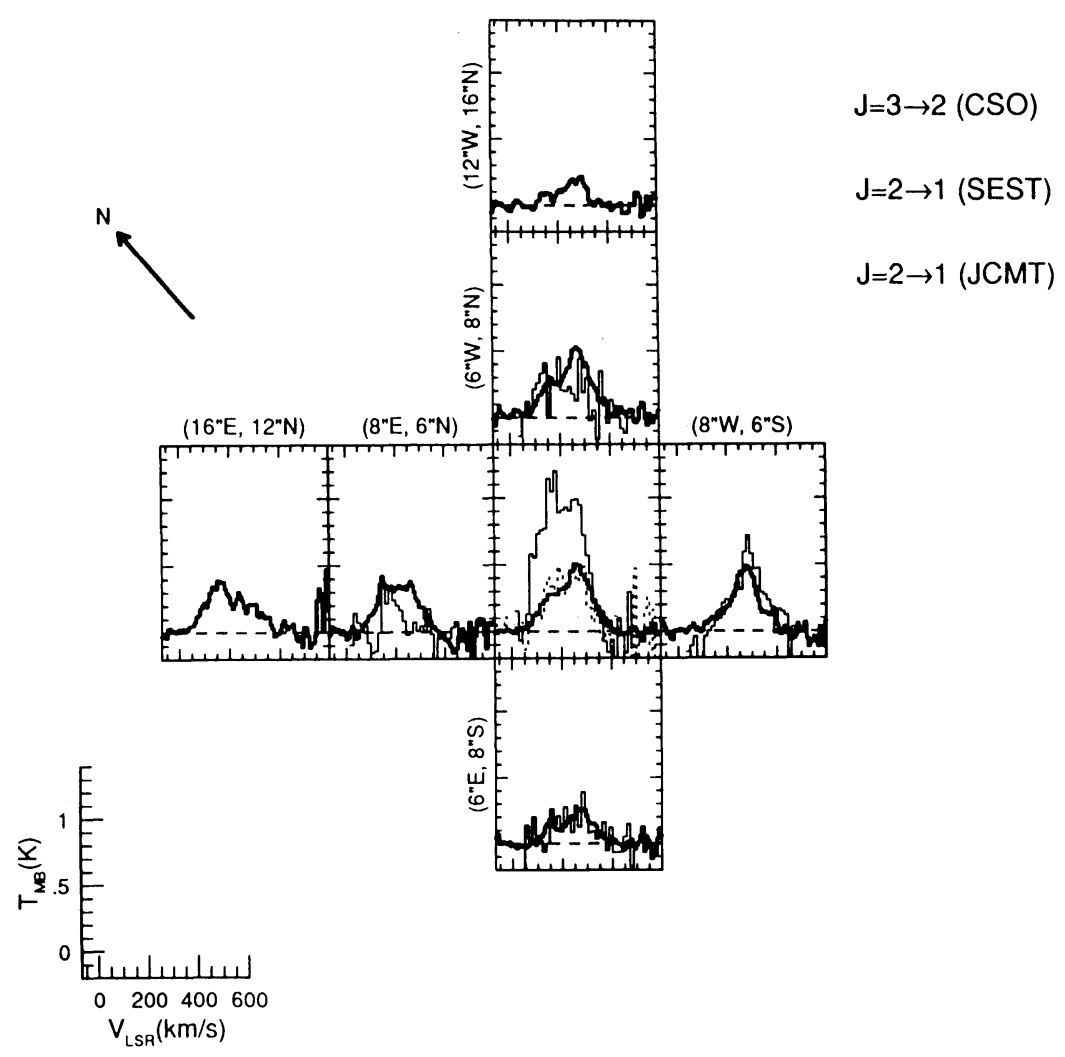

FIG. 3.-The ${ }^{13} \mathrm{CO} J=3 \rightarrow 2$ and $J=2 \rightarrow 1$ spectra, both observed with $20^{\prime \prime}-24^{\prime \prime}$ beams, of NGC 253 are depicted. The spectra are positioned at half-beam spacings along the major axis (horizontal direction in the figure) and minor axis. Notice that the ${ }^{13} \mathrm{CO} J=3 \rightarrow 2$ line intensity is much greater than the ${ }^{13} \mathrm{CO}$ $J=2 \rightarrow 1$ line intensity and declines more rapidly away from the center.

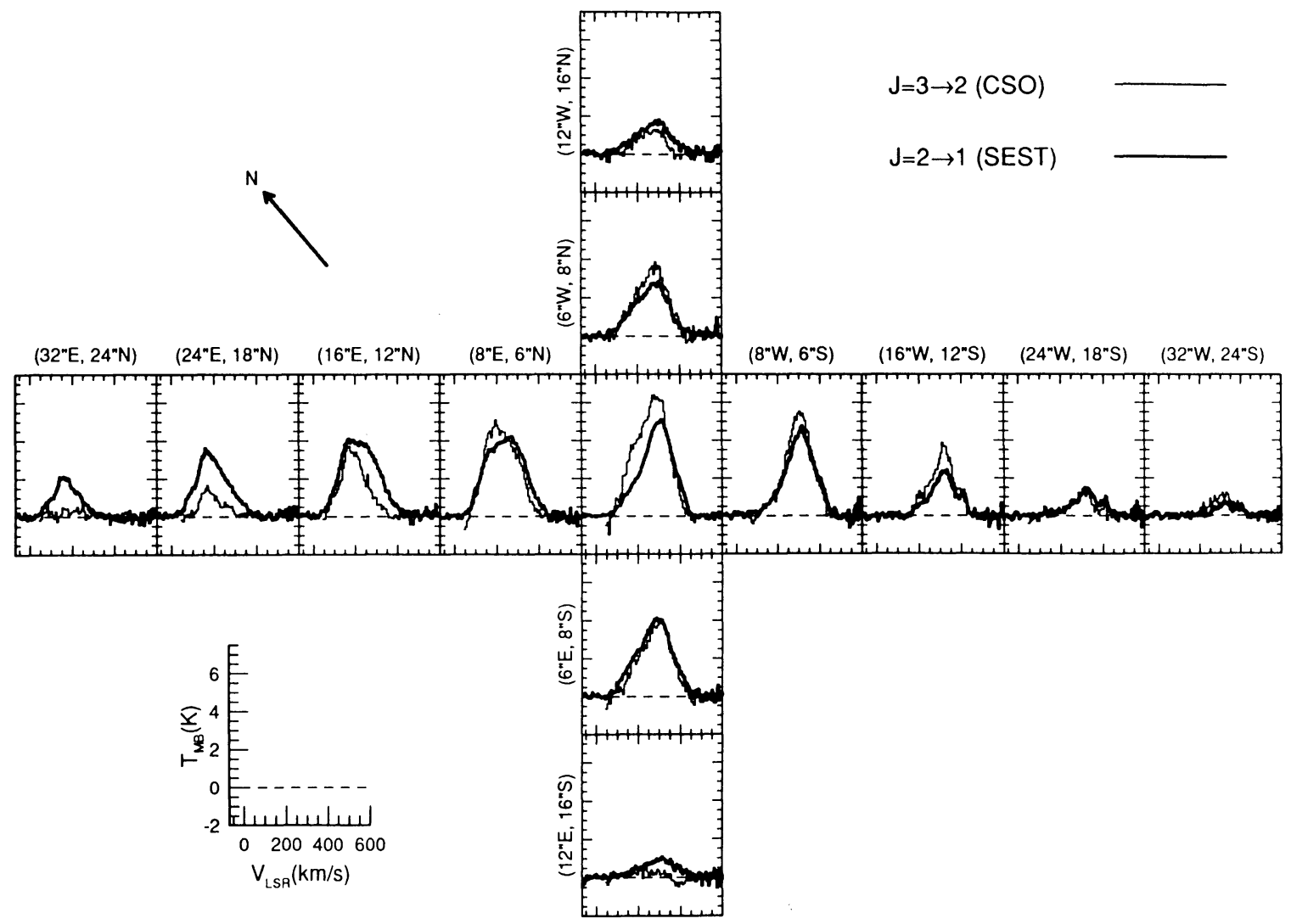

Fig. 4.-The ${ }^{12} \mathrm{CO} J=3 \rightarrow 2$ and $J=2 \rightarrow 1$ spectra, both observed with $21^{\prime \prime}-24^{\prime \prime}$ beams, of NGC 253 are depicted. The spectra are positioned at half-beam spacings along the major axis (horizontal direction in the figure) and minor axis.

\section{(C) American Astronomical Society • Provided by the NASA Astrophysics Data System}


$J=2 \rightarrow 1$ emission implies a strong excitation gradient in the center. There is at least some dense material throughout the central $40^{\prime \prime}$, since the $J=1 \rightarrow 0$ lines of $\mathrm{HCO}^{+}$and $\mathrm{HCN}$ and the $J=2 \rightarrow 1$ line of CS, which have about an order of magnitude higher critical densities than does $\mathrm{CO} J=3 \rightarrow 2$ [i.e., $\left.n_{\text {crit }}(\mathrm{CO} J=3 \rightarrow 2) \sim 5 \times 10^{4} \mathrm{~cm}^{-3}\right]$, arise in an emission region $\sim 3$ times the size of the ${ }^{13} \mathrm{CO} J=3 \rightarrow 2$ emission region (Rieu et al. 1989; Mauersberger \& Henkel 1989).

The smaller spatial extent of the ${ }^{13} \mathrm{CO} J=3 \rightarrow 2$ emission region as well as the differences between the ${ }^{13} \mathrm{CO} J=3 \rightarrow 2$ and $J=2 \rightarrow 1$ line profiles implies that different gas in the nuclear region dominates the emission in each of these lines. As a consequence, the true ${ }^{13} \mathrm{CO} J=3 \rightarrow 2 / J=2 \rightarrow 1$ ratio in the gas where most of the $J=3 \rightarrow 2$ emission arises must be very close to the hot, optically thin limit. Hence, the ${ }^{13} \mathrm{CO}$ $J=3 \rightarrow 2$ emitting gas must be hot $\left(T_{K} \gtrsim 100 \mathrm{~K}\right)$ and thermalized in the $J=3 \rightarrow 2$ line [i.e., $n\left(\mathrm{H}_{2}\right) \gtrsim 5 \times 10^{4} \mathrm{~cm}^{-3}$ ]. Midinfrared continuum fluxes also suggest high temperatures for some of the dust in a region similar in size to the ${ }^{13} \mathrm{CO}$ $J=3 \rightarrow 2$ emission region (i.e., $T_{\text {dust }} \gtrsim 150 \mathrm{~K}$, for $\lambda=10-20$ $\mu \mathrm{m}$ within a 6 " FWHM source toward the center of NGC 253, see Ho et al. 1989). Also, Scoville et al. (1985) argue that the $\lambda=1-2 \mu \mathrm{m}$ colors imply dust temperatures of $600-1000 \mathrm{~K}$ within the central 20" diameter of NGC 253.

Multitransition observations of $\mathrm{HC}_{3} \mathrm{~N}$ provide further evidence for warm molecular gas in the nucleus (Mauersberger et. al. 1990). The $\mathrm{HC}_{3} \mathrm{~N}$ data require $T_{K}=60 \mathrm{~K}$ gas to explain the low-frequency lines $(v<110 \mathrm{GHz})$ and an additional component with $T_{K}=150 \mathrm{~K}$ to explain the high-frequency lines.

The ${ }^{12} \mathrm{CO}$ lines also show evidence for warm molecular gas in the nucleus. The central $30^{\prime \prime}-40^{\prime \prime}$ diameter has ${ }^{12} R_{21}>1$ and ${ }^{12} R_{32} \gtrsim 1$ implying that warm $\left(T_{K} \geq 20 \mathrm{~K}\right)$, not too optically thick $(\tau \lesssim 1)$ gas dominates the emission in these lines. This contrasts with the centers of galaxies IC 342 and M82 where ${ }^{12} R_{32}<1$ (i.e., ${ }^{12} R_{32} \simeq 0.4-0.6$; Wall \& Jaffe 1990; Tilanus et al. 1990) but is comparable to the ${ }^{12} R_{32}$ observed in M83 and Maffei 2 (i.e., ${ }^{12} R_{32} \simeq 1.1-1.3$; Wall et al. 1991a, b). Figure 2 shows the ${ }^{12} \mathrm{CO} J=1 \rightarrow 0, J=2 \rightarrow 1, J=3 \rightarrow 2$ spectra toward NGC 253 where the $J=2 \rightarrow 1$ and $J=3 \rightarrow 2$ data have been smoothed to the $43^{\prime \prime}$ resolution of the $J=1 \rightarrow 0$ data. The brightness of ${ }^{12} \mathrm{CO}$ lines also implies high temperatures in the nuclear region. For instance, at the peak of the ${ }^{12} \mathrm{CO} J=3 \rightarrow 2$ line, $T_{\mathrm{MB}}=6.2 \mathrm{~K}, V_{\mathrm{LSR}}=290 \mathrm{~km} \mathrm{~s}{ }^{-1}$. Material within $\pm 5 \mathrm{~km} \mathrm{~s}^{-1}$ of the peak of the ${ }^{12} \mathrm{CO} J=3 \rightarrow 2$ line (assuming the velocity widths of the individual clouds, $\Delta V_{\text {cloud }}$, to be $=10 \mathrm{~km} \mathrm{~s}^{-1}$, and using the velocity field shown in Canzian et al. 1988) occupies less than $\sim 1 / 10$ of our beam area. Correcting for this velocity field dilution yields $T_{K} \geq 70 \mathrm{~K}$ (since $T_{K} \geq T_{\mathrm{MB}}$ ). Hence, even though the uncertainty in the ${ }^{13} R_{32}$ will allow $T_{K}$ as low as $30 \mathrm{~K}$, the strength of the ${ }^{12} \mathrm{CO}$ $J=3 \rightarrow 2$ line makes this unlikely.

\subsection{Bar Emission}

There is evidence for a decline in excitation with distance from the inner part of the "bar." The ${ }^{12} R_{32}$ values, after shifting the SEST ${ }^{12} \mathrm{CO} J=2 \rightarrow 1$ data by $5^{\prime \prime}$ to the southwest, are $\simeq 1$ at the nucleus and decline to 0.4 and 0.6 at positions $\sim 400$ pc $\left(30^{\prime \prime}\right)$ to the northeast and southwest, respectively. In the LTE limit, the observed ${ }^{12} R_{32}$ 's require the molecular gas temperature to drop to 5-7 K. But the observed Planck brightness temperature is $6 \mathrm{~K}$. Since it is improbable that the gas completely fills the beam at any one velocity, it is likely that non-LTE effects are important in the CO line emission $400 \mathrm{pc}$ from the center and the density must be less than the critical density of the $J=3 \rightarrow 2$ transition $\left[n\left(\mathrm{H}_{2}\right) \lesssim 5 \times 10^{4} \mathrm{~cm}^{-3}\right]$. For the observed positions $10^{\prime \prime}$ from the center, allowing for the contribution of a pointlike nuclear component in ${ }^{13} \mathrm{CO}$ $J=3 \rightarrow 2$, the ${ }^{13} R_{32}$ is significantly less than ${ }^{12} R_{32}$ at the same positions. This difference implies that the ${ }^{12} \mathrm{CO} J=3 \rightarrow 2$ line may be more fully thermalized than the ${ }^{13} \mathrm{CO} J=3 \rightarrow 2$ line.

\section{DISCUSSION}

The CO emission from the central $\sim 1^{\prime}$ diameter of NGC 253 arises predominantly in a $\sim 40^{\prime \prime} \times 10^{\prime \prime}(480 \times 120 \mathrm{pc})$ FWHM barlike region (Canzian et al. 1988) but also comes from a hot, compact central source. The central source has a diameter $\lesssim 15^{\prime \prime}$ and has $T_{K} \gtrsim 100 \mathrm{~K}$ and $n\left(\mathrm{H}_{2}\right) \gtrsim 5 \times 10^{4}$ $\mathrm{cm}^{-3}$, as deduced in $\S 3.1$ from the ratio of ${ }^{13} \mathrm{CO} J=3 \rightarrow 2$ to ${ }^{13} \mathrm{CO} J=2 \rightarrow 1$ line intensities (i.e., ${ }^{13} R_{32}$ ). At positions $30^{\prime \prime}$ from the nucleus along the "bar," the density drops to less than $5 \times 10^{4} \mathrm{~cm}^{-3}$, as deduced from the ratio of ${ }^{12} \mathrm{CO} J=3 \rightarrow 2$ to ${ }^{12} \mathrm{CO} J=2 \rightarrow 1$ line intensities (i.e., ${ }^{12} R_{32}$ ). The picture presented above is insensitive to the calibration uncertainties. Even though the calibration can be uncertain by $20 \%$ or more, the spatial variation of line ratios is much more reproducible. Both the ${ }^{13} \mathrm{CO}$ line ratios and ${ }^{12} \mathrm{CO}$ line ratios imply a drop in excitation away from the nucleus. The picture presented above will be examined in greater detail in the following subsections.

\subsection{Nucleus}

\subsubsection{Column Density and Cloud Size}

We can place limits on molecular cloud dimensions and column densities from the beam-averaged column density and observed ${ }^{13} \mathrm{CO} J=3 \rightarrow 2$ peak brightness temperature. A beam-averaged column density, $\left\langle N\left(\mathbf{H}_{2}\right)\right\rangle$, can be estimated from the ${ }^{13} \mathrm{CO}$ integrated line strengths, assuming an optically thin line in LTE, from the following:

$$
\begin{aligned}
\left\langle N\left(\mathrm{H}_{2}\right)\right\rangle=5.0 & \times 10^{12} T_{K} \frac{\exp \left(31.8 / T_{K}\right)}{X\left({ }^{13} \mathrm{CO}\right)} \\
& \times \int T_{\mathrm{MB}}\left({ }^{13} \mathrm{CO} J=3 \rightarrow 2\right) d V,
\end{aligned}
$$

where $\int T_{\mathrm{MB}}\left({ }^{13} \mathrm{CO} J=3 \rightarrow 2\right) d V$ is in units of $\mathrm{K} \mathrm{km} \mathrm{s}^{-1}$. We have assumed a ${ }^{13} \mathrm{CO} /{ }^{12} \mathrm{CO}$ abundance ratio of $1 / 30$, similar to that observed in the center of our Galaxy (Langer \& Penzias 1990), and so we adopt $X\left({ }^{13} \mathrm{CO}\right)=(1 / 30) \times 10^{-4}$. In NGC 253 , assuming $T_{K}=100 \mathrm{~K}$, we have $\left\langle N\left(\mathrm{H}_{2}\right)\right\rangle \simeq 4 \times 10^{22} \mathrm{~cm}^{-2}$ for the ${ }^{13} \mathrm{CO} J=3 \rightarrow 2$ emitting gas at position $(0,0)$. For a $100 \mathrm{~K}, 15^{\prime \prime}$ diameter ${ }^{13} \mathrm{CO} J=3 \rightarrow 2$ emission region, the area filling factor per velocity interval, $\eta_{\mathrm{ff}}$, is greater than $\sim 0.02$, where the lower limit applies if the line is optically thick. If $\Delta V_{\text {cloud }}=10 \mathrm{~km} \mathrm{~s}^{-1}$ then the clouds at all velocities cover greater than $\sim 0.4$ of the $15^{\prime \prime}$ diameter nuclear region. The clumn density of a typical cloud is then less than $\sim 10^{23} \mathrm{~cm}^{-2}$. For regions with densities comparable to the critical density of ${ }^{13} \mathrm{CO} J=3 \rightarrow 2\left(5 \times 10^{4} \mathrm{~cm}^{-3}\right)$, the typical cloud size is less than $\sim 1 \mathrm{pc}$. The total mass of the molecular gas in the central beam (240 pc diameter) is $\sim 3 \times 10^{7} M_{\odot}$ (applying $\cos i$ to correct to "face-on" column densities is not appropriate since the source is unresolved in both major and minor axis directions). By comparison, the total (stellar plus gas) mass in the central $240 \mathrm{pc}\left(20^{\prime \prime}\right)$ diameter, estimated from the [Ne II] $12.8 \mu \mathrm{m}$ line rotation curve (J. M. Achtermann \& J. H. Lacy 1990 , private communication), is $\sim 2-4 \times 10^{8} M_{\odot}$. 
$\mathrm{HCO}^{+}$observations at $6 "$ resolution (Carlstrom et al. 1990) show that a torus $\sim 10^{\prime \prime}(120 \mathrm{pc})$ in diameter dominates the nuclear emission in NGC 253. Turner (1985) also infers a toroidal distribution from optical depth maps of the $\mathrm{OH} 1665-1667$ $\mathrm{MHz}$ lines. The torus is not directly seen in the ${ }^{12} \mathrm{CO}$ $J=1 \rightarrow 0$ interferometer map of Canzian et al. but may be responsible for the $\sim 2 \times$ higher brightness in the inner $\sim 10^{\prime \prime}$ diameter region.

\subsubsection{Far-UV Heating}

A substantial portion of $\mathrm{CO}$ emission comes from photodissociation regions (PDRs) at cloud edges (Hollenbach 1989). Far-ultraviolet (FUV) radiation from hot stars heats the gas at the cloud edge through ejection of electrons from grains (Hollenbach 1989). The nuclear region of NGC 253 is a copious source of UV photons. $\mathrm{Br} \alpha, \mathrm{Br} \gamma$, and $3 \mathrm{~mm}$ continuum observations are consistent with a Lyman continuum photon flux of $\sim 5 \times 10^{53} \mathrm{~s}^{-1}$ within the central 10" diameter (Beck \& Beckwith 1984; Rieke, Lebofsky, \& Walker 1988; Carlstrom et al. 1990). The nucleus of NGC 253 must also radiate large numbers of the far-UV photons necessary to excite PDRs. The FUV energy density, $G_{0}$ (where $G_{0}=1$ corresponds to the mean interstellar FUV radiation field in our Galaxy), can be estimated either from the number of Lyman continuum photons derived from the $\mathrm{Br} \alpha$ flux by assumming a mean stellar type or from the far-IR luminosity by using equation (6) of Wolfire et al. (1990). Both methods yield $G_{0} \sim 10^{4}$, with the largest uncertainty resulting from assumptions about the spatial relationship of the FUV emitting stars and the molecular clouds. This FUV energy density is similar to that in the Orion Bar (Wolfire, Hollenbach, \& Tielens 1989) and is an order of magnitude more than in the reflection nebula NGC 2023 (Jaffe et al. 1990; van Dishoeck \& Black 1988). Hence, the nuclear FUV emission may heat the gas in the central 10"-20" (120-240 pc) diameter.

One important question is whether the source of the FUV radiation can lie close to the nucleus and inside a molecular torus, like that seem in $\mathrm{HCO}^{+}$or $\mathrm{OH}$, or if the FUV sources must be interspersed with the molecular material. We can use the size and number of clouds emitting ${ }^{13} \mathrm{CO} J=3 \rightarrow 2$ in the nucleus to make this test. The average distance between the clouds along a line of sight, $\lambda$, is given by

$$
\lambda=4\left(n_{c} \pi \mathscr{L}^{2}\right)^{-1}
$$

with $n_{c}$ as the volume density of clouds in units of $\mathrm{pc}^{-3}$ and where $\mathscr{L}$ is the cloud diameter in pc. The quantity $\lambda$ is an attenuation length for the exciting radiation if the intercloud opacity is low and the cloud opacity is high. If $t$ is the thickness of the molecular torus and if $\lambda \ll t$, it is unlikely that sources near the nucleus heat the gas. If $\lambda \gtrsim t$, however, then a central heating source is possible. Adopting a density of $n\left(\mathrm{H}_{2}\right)=10^{5}$ $\mathrm{cm}^{-3}$ yields $1.2 \times 10^{4} \mathscr{L}^{-3}$ as the number of clouds comprising $3 \times 10^{7} M_{\odot}$ of molecular gas in the central beam radius $\left(10^{\prime \prime}=120 \mathrm{pc}\right)$. Assuming the ${ }^{13} \mathrm{CO} J=3 \rightarrow 2$ emission originates in the $\mathrm{HCO}^{+}$torus, the inner radius $(\sim 0 \mathrm{pc})$ and outer radius $(\sim 120 \mathrm{pc})$ implies $n_{c}=2.7 \times 10^{-3} \mathscr{L}^{-3}$, resulting in

$$
\lambda=480 \mathscr{L} \text {. }
$$

Under these assumptions $\lambda / t=1$ for $\mathscr{L}=0.25$. For our typical cloud size of less than $1 \mathrm{pc}$, either nuclear or local heating are then possible. The cloud sizes derived from the $\mathrm{OH}$ observations (i.e., $15 \mathrm{pc}$, Turner 1985) imply $\lambda / t \simeq 60$. The computations above are insensitive to morphology (e.g., $\lambda$ changes by less than a factor of 2 if a spherical emitting region were assumed). The assumption of high cloud-intercloud opacity contrasts $(\gtrsim 100)$ is not unreasonable since [C II] $158 \mu \mathrm{m}$ observations of the sources W3, NGC 1977, and M17SW in our Galaxy also argue for similar contrasts (Howe et al. 1991; Stutzki et al. 1988; Stutzki \& Güsten 1990).

In the nuclear region of NGC 253 , the ${ }^{13} \mathrm{CO} J=3 \rightarrow 2$ and $10 \mu \mathrm{m}$ sources have similar sizes. The ratio of the $10 \mu \mathrm{m}$ flux density to $6 \mathrm{~cm}$ thermal flux density, $S_{10 \mu \mathrm{m}} / S_{6 \mathrm{~cm}}^{\text {th }}$, is 50 (Ho et al. 1989), which is 5 times higher than that found in Galactic and extragalactic $\mathrm{H}$ II regions (e.g., Thronson, Campbell, \& Harvey 1978). This $10 \mu \mathrm{m}$ "excess" led to the suggestion of exotic heating mechanisms for the grains in NGC 253 (Ho et al. 1989). External galaxy observations, however, sample whole clouds, not just $\mathrm{H}$ II regions and their immediate vicinities. Over entire molecular clouds one would expect $S_{10 \mu \mathrm{m}} / S_{6 \mathrm{~cm}}^{\text {th }}$ to be higher than that for the $\mathrm{H}$ II regions themselves. Integrated over the entire Orion B molecular cloud, for instance, the ratio of the IRAS $12 \mu \mathrm{m}$ flux density to the $1.7 \mathrm{GHz}$ flux density (an approximation to $S_{10 \mu \mathrm{m}} / S_{6 \mathrm{~cm}}^{\mathrm{th}}, 1.7 \mathrm{GHz}$ flux density from Barnes et al. 1989) is 150. Similarly, in a region $\sim 1^{\circ}$ west of the peak emission in W3, $S_{10 \mu \mathrm{m}} / S_{6 \mathrm{~cm}}^{\text {th }} \simeq 50$ (see Telesco et al. 1991). Large values of $S_{10 \mu \mathrm{m}} / S_{6 \mathrm{~cm}}^{\mathrm{th}}$ may be typical for GMCs with large-scale PDRs. Hence, the large $S_{10 \mu \mathrm{m}} / S_{6 \mathrm{~cm}}^{\text {th }}$ observed in the nucleus of NGC 253 is consistent with the PDR excitation picture.

While it is possible that the central UV source excites the PDRs in the central $20^{\prime \prime}(240 \mathrm{pc})$ diameter, PDR models suffer the shortcoming that they predict $T_{K} \geq 100 \mathrm{~K}$ for only a few $A_{v}$ into a cloud (Tielens \& Hollenbach 1985). The derived column densities of warm gas, $N\left(\mathrm{H}_{2}\right)=10^{22}-10^{23} \mathrm{~cm}^{-2}$, correspond to $A_{v} \simeq 6-60$, but observations of the ${ }^{13} \mathrm{CO} J=6 \rightarrow 5$ and ${ }^{13} \mathrm{CO} J=3 \rightarrow 2$ lines in Orion (Graf et al. 1990) also require hot $\left(T_{K} \gtrsim 100 \mathrm{~K}\right)$ molecular gas with high column densities $\left[N\left(\mathrm{H}_{2}\right)=3 \times 10^{22}-4 \times 10^{23} \mathrm{~cm}^{-2}\right]$. The current PDR models only account for $\sim 1 / 10$ of the observed hot $\left(T_{K} \gtrsim 100 \mathrm{~K}\right)$ gas. Alternative heating mechanisms (e.g., dust-gas collision, shocks) will not explain the ${ }^{13} \mathrm{CO}$ emission in Orion (Graf et al. 1990), but may be feasible in NGC 253. NGC 253 shows evidence for an active nucleus. The $\mathrm{OH}$ observations also show a "plume" of molecular gas extending 80 " to the north (Turner 1985), while X-ray observations (Fabbiano \& Trinchieri 1984) reveal an outflow $\sim 70^{\prime \prime}$ to the southwest of the nucleus. The [Ne II] $12.8 \mu \mathrm{m}$ line from recent observations with $\sim 1^{\prime \prime}$ resolution show $200 \mathrm{~km} \mathrm{~s}^{-1}$ wide (FWHM) profiles within a 5" FWHM source (J. M. Achtermann \& J. H. Lacy 1990, private communication). The high level of dynamical activity could, in principle, be responsible for some of the excitation of the ${ }^{13} \mathrm{CO}$ $J=3 \rightarrow 2$ emitting clouds.

\subsubsection{Non-LTE CO Excitation?}

As a check of the LTE excitation analysis toward the nucleus, we have examined a grid of non-LTE models based on the large velocity gradient hereafter (LVG; see de Jong et al. 1975) scheme and compared them to the observations. Subthermal excitation and radiative trapping may affect physical conditions derived assuming LTE. In general, the physical conditions derived from LVG models matching the observed line ratios were close to those derived assuming LTE. We considered an LVG model an acceptable fit to the observations when the model line ratios were within $30 \%$ of the observed ratios. In addition, the model radiation temperatures had to exceed the observed radiation temperatures because the gas 
would not necessarily fill the beam. (In reality, we only required the model radiation temperature to exceed 0.8 of the observed radiation temperature to account for calibration uncertainties.) The LVG models suggest that the kinetic temperature and molecular gas density are $T_{K} \geq 60 \mathrm{~K}$ (and more likely $>100 \mathrm{~K}$ as implied by the LTE calculation) and $n\left(\mathrm{H}_{2}\right) \gtrsim$ $10^{4}-10^{5} \mathrm{~cm}^{-3}$. Assuming $\Delta V_{\text {cloud }}=10 \mathrm{~km} \mathrm{~s}^{-1}$ for the individual clouds in NGC 253, the column density is $N\left(\mathrm{H}_{2}\right) \simeq$ $10^{22}-10^{23} \mathrm{~cm}^{-2}$. These values are consistent with our previous estimates.

\subsection{Bar Emission}

\subsubsection{Temperature and Density}

The density and temperature in the circumnuclear ${ }^{12} \mathrm{CO}$ $J=1 \rightarrow 0$ "bar" is significantly lower than in the nuclear molecular gas. The low ${ }^{13} R_{32}(<0.7)$ and high ${ }^{12} R_{32}(\geq 1)$ outside the central $20^{\prime \prime}(240 \mathrm{pc})$ diameter requires densities lower than these critical densities, suggesting that the proportion of high density gas is smaller than at the central position. The size of the ${ }^{13} \mathrm{CO} J=3 \rightarrow 2$ line emission region, which is smaller than the region emitting in the lines of high dipole moment (i.e., high critical density) molecules such as $\mathrm{HCN}$, $\mathrm{HCO}^{+}$, and $\mathrm{CS}$ is the main piece of evidence for the temperature drop. In $\sim 20^{\prime \prime}$ beams, $\mathrm{HCN}, \mathrm{HCO}^{+} J=1 \rightarrow 0$ and CS $J=2 \rightarrow 1$ observations show a source size of $\sim 40^{\prime \prime}(=480$ pc) FWHM (Rieu et al. 1989, Mauersberger \& Henkel 1989) as compared to the $\lesssim 15^{\prime \prime}(=180 \mathrm{pc})$ FWHM ${ }^{13} \mathrm{CO} J=3 \rightarrow 2$ source size. The $J=1 \rightarrow 0$ lines of $\mathrm{HCN}$ and $\mathrm{HCO}^{+}$, the $J=2 \rightarrow 1$ line of $\mathrm{CS}$, and the ${ }^{13} \mathrm{CO} J=3 \rightarrow 2$ line all have critical densities greater than $10^{4}-10^{5} \mathrm{~cm}^{-3}$. However, since the ${ }^{13} \mathrm{CO} J=3 \rightarrow 2$ line strength decreases inside the region of strong $\mathrm{HCN}, \mathrm{HCO}^{+}, \mathrm{CS}$ emission, the kinetic temperature must also decline with increasing distance from the center. The ${ }^{13} \mathrm{CO} J=3 \rightarrow 2$ line is more temperature sensitive than the lower $J$ lines of the high dipole moment molecules because the ${ }^{13} \mathrm{CO} J=3$ level is $32 \mathrm{~K}$ above ground, whereas the $\mathrm{CS} J=2$ state is only $7 \mathrm{~K}$ above ground and the $J=1$ states of $\mathrm{HCN}$ and $\mathrm{HCO}^{+}$are only $4 \mathrm{~K}$ above ground. It follows that the ${ }^{13} \mathrm{CO} J=3 \rightarrow 2$ line will be stronger in the hotter parts of the CS $J=2 \rightarrow 1, \mathrm{HCN}, \mathrm{HCO}^{+} J=1 \rightarrow 0$ emitting region. In going from $T_{K}=100 \mathrm{~K}$ to $T_{K}=10 \mathrm{~K}$, the ${ }^{13} \mathrm{CO} J=3 \rightarrow 2$ line strength decreases 5-6 times relative to the strength of these $\mathrm{HCO}^{+}, \mathrm{HCN}$, and $\mathrm{CS}$ lines at constant density and column density. The emission from high dipole moment molecules in the "bar" where ${ }^{13} \mathrm{CO} J=3 \rightarrow 2$ is undetectable implies that the "bar" has a kinetic temperature significantly lower than the greater than $\sim 100 \mathrm{~K}$ gas temperature in the nucleus. The measured ${ }^{12} R_{32}$ values in the "bar" support this conclusion since they imply temperatures of $10-40 \mathrm{~K}$. This temperature difference argues for additional heating sources in the inner $10^{\prime \prime}$ of NGC 253. There is good observational evidence for the existence of such sources. The extinction-corrected $\mathrm{Br} \alpha$ flux decreases by factors of $\sim 3$ or more outside the central $6^{\prime \prime}$ (70 pc) diameter (Beck \& Beckwith 1984), the [Ne II] $12.8 \mu \mathrm{m}$ emission has a size of $5^{\prime \prime} \times 3^{\prime \prime}$ FWHM (J. M. Achtermann \& J. H. Lacy 1990, private communication), and the deconvolved 3 $\mathrm{mm}$ continuum emission is $\sim 10^{\prime \prime} \times 4^{\prime \prime}$ FWHM in size (Carlstrom et al. 1990) implying that the source(s) of Lyman continuum photons is strongly centrally peaked.

Indications of predominantly low density gas $\left[n\left(\mathrm{H}_{2}\right) \lesssim\right.$ $10^{4}-10^{5} \mathrm{~cm}^{-3}$ ] are not restricted to the inner part of the bar; ${ }^{12} R_{32}$ at positions $30^{\prime \prime}$ along the major axis also imply low molecular gas densities. Thirty arcseconds northeast along the major axis [position $\left(24^{\prime \prime}, 18^{\prime \prime}\right)$ ], for example, the LVG models (allowing for the 30\% uncertainties in the line ratios) require $n\left(\mathrm{H}_{2}\right) \lesssim 3 \times 10^{3} \mathrm{~cm}^{-3}$ and $N\left(\mathrm{H}_{2}\right) \lesssim 10^{23} \mathrm{~cm}^{-2}$ for gas warmer than $10 \mathrm{~K}$. Alternatively, the density could be as high as $10^{4}$ $\mathrm{cm}^{-3}$ if the molecular gas is cold (i.e., $T_{K} \leq 10 \mathrm{~K}$ ).

\subsubsection{Mass}

The molecular "bar" contains a large amount of neutral interstellar material. The mass of molecular gas in the central $40-50^{\prime \prime}$ diameter can be estimated using the ${ }^{13} \mathrm{CO} J=2 \rightarrow 1$ integrated line strengths at the central five positions observed. The kinetic temperatures adopted for the positions $\left(+8^{\prime \prime},+6^{\prime \prime}\right)$, $\left(-8^{\prime \prime},-6^{\prime \prime}\right),\left(-6^{\prime \prime},+8^{\prime \prime}\right)$, and $\left(+6^{\prime \prime},-8^{\prime \prime}\right)$ range from 20 to $40 \mathrm{~K}$ based on the ${ }^{12} R_{32}$ values at these positions. The derived column densities are corrected to "face-on" values by multiplying by a factor of $\cos i(=0.2)$. Averaged over the central $480-600 \mathrm{pc}\left(40-50^{\prime \prime}\right)$ diameter and including the central source (where the cos $i$ factor was not applied, see $\S 4.1$ ), the face-on column density is $\sim 1 \times 10^{22} \mathrm{~cm}^{-2}$. This compares with the $\left\langle N\left(\mathrm{H}_{2}\right)\right\rangle=5 \times 10^{21} \mathrm{~cm}^{-2}$ in the central $55^{\prime \prime}$ derived from 350 $\mu \mathrm{m}$ observations (Gear et al. 1986). [Devereux \& Young (1990) find that column densities estimated from far-IR dust emission can be $\sim 10$ times too low, especially if the dust temperature is determined from IRAS $60 \mu \mathrm{m}$ to $100 \mu \mathrm{m}$ flux ratios. This flux ratio typically gives dust temperatures of $30-40 \mathrm{~K}$ for galactic disks, and using $T_{\text {dust }}=20 \mathrm{~K}$ yields better results. However, the Devereux and Young results apply to entire galactic disks, and column densities derived from $350 \mu \mathrm{m}$ observations are less temperature sensitive than those derived from $100 \mu \mathrm{m}$ observations. Even if we use $T_{\text {dust }}=20 \mathrm{~K}$, instead of the $40 \mathrm{~K}$ adopted by Gear et al., we obtain $N\left(\mathrm{H}_{2}\right)=2 \times 10^{22} \mathrm{~cm}^{-2}$, still within a factor 2 of our ${ }^{13} \mathrm{CO} J=2 \rightarrow 1$ derived column densities. Also, the 86 and $151 \mu \mathrm{m}$ fluxes (Telesco \& Harper 1980) for the central 50" diameter of NGC 253 imply $T_{\text {dust }}=30-40 \mathrm{~K}$.] The total molecular gas within a $\sim 500 \mathrm{pc}$ diameter is $\sim 4 \times 10^{7}$ $M_{\odot}$. The whole $\sim 100^{\prime \prime} \times 60^{\prime \prime}$ region observed in ${ }^{12} \mathrm{CO}$ $J=2 \rightarrow 1$ contains $\sim 5 \times 10^{7} M_{\odot}$ of molecular gas (assuming a ${ }^{13} \mathrm{CO} J=2 \rightarrow 1 /{ }^{12} \mathrm{CO} J=2 \rightarrow 1$ integrated intensity ratio of 0.1 throughout the "bar").

\subsection{Small-Scale Inhomogeneities}

The existence of inhomogeneities on scales smaller than a beam (cold or diffuse gas present in regions where warm gas dominates the line emission or vice versa) will not change the basic picture of the molecular gas conditions in NGC 253: a predominantly hot $\left(T_{K} \gtrsim 100 \mathrm{~K}\right)$, dense $\left[n\left(\mathrm{H}_{2}\right) \gtrsim 5 \times 10^{4}\right.$ $\mathrm{cm}^{-3}$ ) nuclear region and a predominantly cooler $\sim 40^{\prime \prime} \times 10^{\prime \prime}$ bar with lower densities at its ends [e.g. 30" northeast of the nucleus $\left.n\left(\mathrm{H}_{2}\right) \lesssim 3 \times 10^{3} \mathrm{~cm}^{-3}\right]$. Cold molecular gas in LTE with $T_{K}=10 \mathrm{~K}$ and $\tau \ll 1$ in the observed ${ }^{13} \mathrm{CO}$ observed lines can contribute no more than $20 \%$ to the mass of the molecular gas in the nucleus of NGC 253. Cold molecular gas that is optically thick in the ${ }^{13} \mathrm{CO} J=2 \rightarrow 1$ and $J=3 \rightarrow 2$ lines, however, could still contribute significantly to the molecular gas mass in the nucleus without affecting the observed ${ }^{13} R_{32}$. Observations of $\mathrm{C}^{18} \mathrm{O}$ lines would test unambiguously for the existence of such optically thick gas.

Even if some higher temperature or higher density gas is present near the ends of the bar, low density $\left[n\left(\mathrm{H}_{2}\right) \lesssim 3 \times 10^{3}\right.$ $\left.\mathrm{cm}^{-3}\right]$ molecular gas must still dominate the ${ }^{12} \mathrm{CO} J=2 \rightarrow 1$ and ${ }^{12} \mathrm{CO} J=3 \rightarrow 2$ line emission at $\left(24^{\prime \prime}, 18^{\prime \prime}\right)$ because of the low observed ${ }^{12} R_{32}$. A cloud ensemble including warmer or denser gas (assuming LTE excitation) cannot explain the low 
observed ${ }^{12} R_{32}$ without either both ${ }^{12} \mathrm{CO}$ lines being weaker than observed or requiring ${ }^{12} R_{21}$ to be much lower than observed. Dense $\left[n\left(\mathrm{H}_{2}\right) \lesssim 5 \times 10^{4} \mathrm{~cm}^{-3}\right]$ gas that contributes a minor portion of the ${ }^{12} \mathrm{CO} J=2 \rightarrow 1$ and ${ }^{12} \mathrm{CO} J=3 \rightarrow 2$ emission cannot be ruled out, however, if its area filling factor per velocity interval is small. Observations of ${ }^{13} \mathrm{CO}$ lines would place limits on the mass that such dense gas contributes to the molecular gas at this position in NGC 253.

\subsection{Comparison with $M 82$}

In addition to having similar far-IR luminosities, NGC 253 is similar to M82 in a number of ways:

1. Both galaxies emit strongly in the lines of ${ }^{12} \mathrm{CO}$ and ${ }^{13} \mathrm{CO}$ (see Tilanus et al. 1990 and references therein for all mentions of M82 in this section), implying that molecular lines play an important role in the energetics of the ISM in the central few hundred parsecs.

2. Both galaxies have $T_{\mathrm{MB}}\left({ }^{12} \mathrm{CO} J=2 \rightarrow 1\right)>T_{\mathrm{MB}}\left({ }^{12} \mathrm{CO}\right.$ $J=1 \rightarrow 0$ ) (i.e., ${ }^{12} R_{21}>1$ ), suggesting the presence of a warm $\left(T_{K} \gtrsim 20 \mathrm{~K}\right)$, optically thin (in these ${ }^{12} \mathrm{CO}$ lines) component of molecular gas.

3. Both exhibit the presence of a gas torus in some lines (e.g., Nakai et al. 1987; Carlstrom et al. 1990) which has a $\sim 400$ pc diameter in M82 and $\sim 120$ pc diameter in NGC 253.

The ${ }^{13} \mathrm{CO} J=3 \rightarrow 2$ line intensity in the central $250 \mathrm{pc}$ of NGC 253 is a factor of greater than $\sim 2$ stronger than that in a similar size region in M82. The ${ }^{13} R_{32}$ climbs to 2 in NGC 253 but is 1 or less in M82. The higher ${ }^{13} R_{32}$ value in the nucleus of NGC 253 suggests higher densities in the bulk of the molecular gas than in M82.

\section{CONCLUSIONS} data:

We conclude the following from our ${ }^{13} \mathrm{CO}$ and ${ }^{12} \mathrm{CO}$ line
1. Most of the ${ }^{13} \mathrm{CO} J=3 \rightarrow 2$ line emission originates in a $\lesssim 15^{\prime \prime}(180 \mathrm{pc})$ source while the $40^{\prime \prime} \times 10^{\prime \prime}$ FWHM barlike feature observed by Canzian et al. (1988) dominates the emission in the other CO lines.

2. ${ }^{13} R_{32} \simeq 2$ implying warm $\left(T_{K} \gtrsim 100 \mathrm{~K}\right)$, dense $\left[n\left(\mathrm{H}_{2}\right) \gtrsim\right.$ $\left.10^{4}-10^{5} \mathrm{~cm}^{-3}\right]$ for the bulk of the molecular gas within the central $\sim 20 "$ ( $240 \mathrm{pc})$.

3. Far-UV heating of PDRs by a central source or by distributed OB stars can warm the molecular gas sufficiently to explain the observed temperatures of the nuclear component. Alternatively, dynamical heating is, in principle also possible.

4. The molecular gas outside the central $20^{\prime \prime}(240 \mathrm{pc})$ diameter region is both cooler and less dense than inside this region, as determined from spatial variation of the ${ }^{13} \mathrm{CO}$ $J=3 \rightarrow 2$ intensity relative to that of ${ }^{13} \mathrm{CO} J=2 \rightarrow 1$ and to the lines of high dipole moment molecules (e.g., CS, $\mathrm{HCO}^{+}$, HCN, Mauersberger \& Henkel 1989; Rieu Nakai, \& Jackson 1989).

5. The density of molecular gas at a position $\gtrsim 30^{\prime \prime}$ to the northeast of the nucleus is probably $\leq 10^{4} \mathrm{~cm}^{-3}$ to account for the low ${ }^{12} R_{32}$ observed.

6. $M\left(\mathrm{H}_{2}\right) \approx 4 \times 10^{7} M_{\odot}$ for the central $40^{\prime \prime}-50^{\prime \prime}(500-600$ pc) diameter.

We thank J. M. Achtermann and J. H. Lacy for sharing their [Ne II] data with us prior to publication. We also thank Neal Evans, Yangsheng Wang, \& Rene Plume for helping with the observations. The CSO is operated by the California Institute of Technology under funding from the National Science Foundation, Grant No. AST-88-15132. This work was supported in part by NSF Grant No. AST-88-15801 to the University of Texas at Austin and by a grant from the W. M. Keck Foundation. Support was also provided by a Texas Advanced Research Project Grant. D. J. acknowledges support from a David and Lucile Packard Foundation Fellowship.

\section{REFERENCES}

Barnes, P. J., Crutcher, R. M., Bieging, J. H., Storey, J. W. V., \& Willner, S. P. 1989 ApJ, 342, 883

Beck, S. C., \& Beckwith, S. V. 1984, MNRAS, 207, 671

Becklin, E. E., Fomalont, E. B., \& Neugebauer, G. 1973, ApJ, 181, L27

Canzian, B., Mundy, L. G., \& Scoville, N. Z. 1988, ApJ, 333, 157

Carlstrom, J. E., Jackson, J. E., Ho, P. T. P., \& Turner, J. L. 1990, in The Interstellar Medium in External Galaxies, ed. D. J. Hollenbach \& H. A. Thronson (Washington, DC: NASA CP 3084), 337

Davidge, T. J., \& Pritchett, C. J. 1990, AJ, 100, 1

de Jong, T., Chu, S. I., \& Dalgarno, A. 1975, ApJ, 199, 69

de Vaucouleurs, G. 1978, ApJ, 224, 79

Devereux, N. A., \& Young, J. S. 1990, ApJ, 359, 42

Ellison, B. N., Schaffer, P. L., Schaal, W., \& Vail, D. 1989, preprint

Fabbiano, G., \& Trinchieri, G. 1984, ApJ, 286, 491

Gear, W. K., Gee, G., Robson, E. I., Ade, P. A. R., \& Duncan, W. D. 1986, MNRAS, 219, 19P

Graf, U. U., Genzel, R., Harris, A. I., Hills, R. E., Russell, A. P. G., \& Stutzki, J. 1990, ApJ, 358, L49

Hildebrand, R. H., Loewenstein, R. F., Harper, D. A., Orton, G. S., Keene, J., \& Whitcomb, S. E. 1985, Icarus, 64, 64

Ho, P. T. P., Turner, J. L., Fazio, G. G., \& Willner, S. P. 1989, ApJ, 344, 135

Hollenbach, D. J. 1989, IAU Symp. 135, Interstellar Dust, ed. A. G. G. M. Tielens \& L. Allamandola (Dordrecht: Kluwer Academic), 227

Howe, J. E., Jaffe, D. T., Genzel, R., \& Stacey, G. J. 1991, ApJ, 374, 158

Jaffe, D. T., Genzel, R., Harris, A. I., Howe, J. E., Stacey, G. J., \& Stutzki, J. 1990, ApJ, 353, 193

Langer, W. D., \& Penzias, A. A. 1990, ApJ, 357, 477

Mauersberger, R., \& Henkel, C. 1989, A\&A, 223, 79

Mauersberger, R., Henkel, C., \& Sage, L. J. 1990, A\&A, 236, 63

Nakai, N., Hayashi, M., Handa, T., Sofue, Y., Hasegawa, T., \& Sasaki, M. 1987, PASJ, 39, 685

Pence, W. D. 1981, ApJ, 247, 473

Rickard, L. J, Palmer, P., Morris, M., Turner, B. E., \& Zuckerman, B. 1977a, ApJ, 213, 673

Rickard, L. J, Palmer, P., Turner, B. E., Morris, M., \& Zuckerman, B. 1977b, ApJ, 214, 390

Rieke, G. H., Lebofsky, M. J., Thompson, R. I., Low, F. J., \& Tokunaga, A. T. 1980, ApJ, 238, 24

Rieke, G. H., Lebofsky, M. J., \& Walker, C. E. 1988, ApJ, 325, 679

Rieu, N.-Q., Nakai, N., \& Jackson, J. M. 1989, A\&A, 220, 57

Scoville, N. Z., Soifer, B. T., Neugebauer, G., Young, J. S., Matthews, K., \& Yerka, J. 1985, ApJ, 289, 129

Stark, A. A., \& Wolff, R. S. 1979, ApJ, 229, 118

Stutzki, J., Stacey, G. J., Genzel, R., Harris, A. I., Jaffe, D. T., \& Lugten, J. B. 1988, ApJ, 332, 379

Stutzki, J., \& Güsten, R. 1990, ApJ, 356, 513

Telesco, C. M., Campins, H., Joy, M., Dietz, K., \& Decher, R. 1991, ApJ, 369, 135

Telesco, C. M., \& Harper, D. A. 1980, ApJ, 235, 392

Thronson, H. A., Campbell, M. F., \& Harvey, P. M. 1978, AJ, 83, 1581

Tielens, A. G. G. M., \& Hollenbach, D. J. 1985, ApJ, 291, 722

Tilanus, R. P. J., Tacconi, L. J., Zhou, S., Sanders, D. B., Sutton, E. C., Lo,

K. Y., Stephens, S. A., \& Wynn-Williams, C. G. 1991, ApJ, in press

Turner, B. E. 1985, ApJ, 299, 312

van Dishoeck, E. F., \& Black, J. H. 1988, ApJ, 334, 771

Wall, W. F., \& Jaffe, D. T. 1990, ApJ, 361, L45

Wall, W. F. et al. 1991a, in preparation

. 1991b, in preparation

Wolfire, M. G., Hollenbach, D., \& Tielens, A. G. G. M. 1989, ApJ, 344, 770

Wolfire, M. G., Tielens, A. G. G. M., \& Hollenbach, D. 1990, ApJ, 358, 116

Wright, E. L. 1976, ApJ, 210, 250

Wynn-Williams, C. G., Becklin, E. E., Mathews, K., \& Neugebauer, G. 1979, MNRAS, 189, 163

Young, J. S., \& Scoville, N. Z. 1984, ApJ, 287, 153 